\title{
Spectacle Retentive Orbital Prosthesis
}

\author{
${ }^{a}$ Ahmed Amin Moselhy, DDS, MS, Phd A, and ${ }^{\mathrm{b}}$ Nasser Aly, DDS, MS, Phd
}

\begin{abstract}
Maxillofacial prosthetics include all the artificial prosthesis that restores missing parts of the face due to trauma, congenital defects or surgically removed as a resolution of tumors.The loss of the facial structures can have a physical, social and psychological blow to those affected.This passing is more pronounced when the affected part is the eye and all orbital contents resulting in gross mutilation.Here we report a case of 55-year-old male patient with retinoblastoma of the right eye, undergone surgical enucleation and was rehabilitated by the spectacle retained silicon orbital prosthesis in order to achieve the perfect fit and aesthetics.
\end{abstract}

Keywords: retinoblastoma, orbital rehabilitation prosthesis, spectacle, room temperature vulcanized silicone

\section{Introduction}

Acquired facial defects are frequently imparted with extensive mutilation, disability, social response to the functional damage and psychological strain.Rehabilitation of maxillofacial defects plays an indispensable function in treatment success,

This passing is more pronounced when the affected part is the eye and all orbital contents, resulting in gross mutilation. When the entire content of the orbit (including muscle fascia, eyelids, conjunctiva and the lacrimal apparatus) is removed, the artificial replacement is referred as an orbital prosthesis. ${ }^{1}$ Success in maxillofacial prosthetics depends on the full cognizance of the principles that underlie facial harmony, color matching, anchorage and retention, weight bearing and leverage, durability and strength of materials used, tissue compatibility and tolerance. The restoration of orbital defects presents a challenge in maxillofacial prosthetics because the ocular prosthesis is enclosed within the orbital prosthesis. ${ }^{2}$ Many variations exist in techniques and materials for fabricating an orbital prosthesis. ${ }^{3}$ Careful positioning of the ocular portion of the orbital prosthesis is one of the requirements for a successful esthetic result. A change of this position, which may occur during fabrication or may be due to the distortion of the prosthetic housing or loss of retention of the prosthesis, may result in an unsatisfactory appearance. ${ }^{2}$ Different methods and materials for fabrication of the orbital prosthesis are available and also many varieties of way in retaining the prosthesis. Retention of orbital prosthesis can be achieved by tissue undercuts, attaching it to different parts of a spectacle, use of adhesives or use of osseointegrated implants. ${ }^{4,5}$

This article presents a simplified technique for fabricating an orbital silicon prosthesis retained by the spectacle.

${ }^{a}$ Lecturer in military medical academy. Department of Maxillofacial Prosthodontic, Kopry El Koba military hospital, Egypt

${ }^{\mathrm{b}}$ Lecturer in Faculty of Oral and Dental Medicine, Nahda University, Egypt

\section{Case Report}

A 55-year-old male with an orbital defect (Fig.1) As a consequence of surgical removal of retinoblastoma was referred to the Department of Prosthodontics at Kobry El Kobba Military Hospital, Cairo, Egypt, for the successor of his exterminated right eye.From the history of the patient, it reveals that he has undergone surgery with extermination of orbit which had been carried out one year ago to eradicate tumour. The defect lined with skin graft, the patient refused to undergo surgical interference to install implants to retain the orbital prosthesis. Therefore spectacle retained orbital prosthesis was planned for the patient to get to him socially presentable.We explained the clinical procedures to the patient before fabrication of the prosthetic device.The patient was motivated and consented to rehabilitate his defect.

The impression for the facial mileage was made with a thin mixture of alginate (irreversible hydrocolloid) (Fig. 2). The area was boxed with modelling wax and then irreversible hydrocolloid (AlginateDentsply) was mixed and painted smoothly into the defect part and also above the closed left eye.The hydrocolloid was reinforced with with thin mix of quick set plaster with open gauze pads, which help in the retention of the Plaster of Paris backing. After removal, the impression is cleaned and inspected for accuracy and detail. Type III stone is poured into the defect and the remaining areas and a cast is obtained.

Master cast was duplicated (Fig. 3).Orientation points are noted along the master roll (Fig.4). Measurements were made from the patient's facial midline to the center of the pupil and from the inner canthus of the eye to the nasal bridge. ${ }^{3}$ Modelling wax was placed on it and tried in the patient's face (Fig.5),to construct 
a permanent base.It was processed by using transparent heat cure acrylic polymerizing resin. It was then tried in patients face. The foundation helped to join the silicone prosthesis to the spectacle of the eyewear (Fig. 6).

A suitable acrylic resin ocular prosthesis, with the same color of its iris/pupil complex, dimensions, and sclera similar to the contralateral eye, was selected and adapted in the anophthalmic area of the working cast with the baseplate wax. The ocular prosthesis was seated and roughly oriented in the defect, according to the orientation marks. This wax pattern was transferred to the patient's anophthalmic area, and the patient was instructed to look straight.The stock ocular prosthesis was adjusted anteroposteriorly, mediolaterally, and superioinferiorly in accordance with the contralateral eye. After sculpting the eyelids, modeling wax was added to the defect side of the face and sculpted by comparing the normal contralateral side of the expression. Final surface contour and skin texture were established by carving in lines and wrinkles observed around the normal eye. Once that position was established remaining portion was sculpted in wax and tried in the patient to maintain the optimum position of the ocular portion inside the orbital prosthesis. During fabrication the back of the ocular piece was notched to ensure that it will not move during flasking and packing of silicone. Acrylic frame was tried with the Spectacle (Fig. 7). Approval from the patient about the look of the orbital prosthesis was achieved. The wax sculpted orbital prosthesis was flasked and dewaxed. ) Over the acrylic base, primer was applied for bonding and separating medium was applied to the mould. Room temperature vulcanizing (RTV) medical-graded silicone material was mixed according to instructions given by the producer.Intrinsic color pigments were added and mixed to achieve the nuance of the skin color of the contralateral side of the expression (Fig. 8).Silicone material was taken and kept for polymerization for $24 \mathrm{~h}$ in a bench press (Fig.9). After $24 \mathrm{~h}$, molds were separated, the silicone prosthesis retrieved, and finishing done. Extrinsic color was used to achieve the perfect color match of the patient's skin and personal characters(Fig. 10). Eyelashes were embedded in the eyelids by the aid of syringe using the patient's own hair (Fig. 11). Select a well fitted, comfortable frame that blends with the personality of the patient and adequately covers the defect when viewed from the front, the sides, and above. The frame was tried in the patient. The prosthesis was held in place in the orbital defect, and the patient was asked to wear the spectacles over the prosthesis. The prosthesis was attached to the spectacle framework by the aid of pins drilled in the acrylic frame (Fig. 12).Lastly, home care instructionswere specified to the patient, follow-up was carried away. The patient should be held at least weekly for the first month and then was after 1, 3, 6, 9, and 12 months.

\section{Discussion}

The replacement of the facial defect and lost eye promotes physical and psychological well-being. ${ }^{6}$ An accurate alignment of the artificial eye plays a major part in the achiever of the orbital prosthesis. The esthetics achieved at the conclusion of the treatment depend on the quantity of tissue removed, good contour of the inferior margin and minimal sagging due to the weightiness of the prosthetic device

In this case we are able to achieve aesthetic and comfort by treating with silicone orbital prosthesis. The option of using acceptable maxillofacial prosthetic material and sensible retentive aid should be the objective of rehabilitating these patients. . Silicone has improved marginal adaptation and realistic appearance, with desirable material properties including flexibility, biocompatibility, ability to accept intrinsic and extrinsic colorants, chemical and physical inertness and mouldability. ${ }^{7}$ Silicone elastomers also are characterized by excellent heat stability and are chemically inert, particularly in body tissues. ${ }^{8,9}$ Flexibility of silicone becomes advantageous when the defect includes movable soft tissue. Silicones can be well treated, cleaned and molded to give a texture and appearance closely simulating skin. ${ }^{4}$

Several methods of retention of a facial prosthesis are eye patches, spectacles, magnets, adhesives, or a compounding of those and osseointegrated implants. Although osseointegrated implants provide superior retention, the patient's unwillingness for another surgery. The most commonly used conventional methods to retain orbital prostheses is the eyeglass frames and anatomic retentive undercuts. ${ }^{10}$. Modelling wax and medical grade silicone were used in the fabrication of orbital prosthesis. Compare to the acrylic prosthesis, silicone orbital prosthesis have some advantages like light weight, better aesthetics. The option of using acceptable maxillofacial prosthetic material and sensible retentive aid should be the objective of rehabilitating these patients.Silicone has improved marginal adaptation and realistic appearance, then it has been utilized for the fabrication of orbital prosthesis. 11 As a rule all orbital prosthesis is supplied with frames to reach the borders of the prosthesis less obvious and attract attention away from the prosthesis. Graduated lenses from dark to clear should be incorporated as this can alleviate any slight color imbalance and decrease metamerism.

\section{Conclusion}

The goal of any prosthetic treatment is to return the patient to society with a normal appearance of the prosthetic eye. This paper elaborates the technique of fabrication of spectacle retained orbital prosthesis for a patient whose eye was exterminated as a result of retinoblastoma of the right eye. Natural appearance depends on holding the completed prosthesis in its proper relation to the head. 
The article shows the steps utilized in making up an orbital prosthesis. Major considerations such as selection of the spectacle frame and the artificial eye, eye alignment, and mold fabrication are emphasized to ensure an esthetically acceptable prosthesis. A simple and cost efficient technique for assembly of the silicone orbital prosthesis was described.

\section{References}

[1]. Joneja OP, Madan SK, Mehra MD, Dogra RN. Orbital prostheses. J Prosthet Dent 1976;36(3):306-11

[2]. Shifman A, Levin AC, Levy M, Lepley JB. Prosthetic restoration of orbital defects. J Prosthet Dent 1979;42(5):543-6.

[3]. Mekayarajjananonth T, Salinas TJ, Chambers MS, Lemon JC. A mold-making procedure for multiple orbital prostheses fabrication. J Prosthet Dent 2003;90(1):97-100

[4]. Glantz P O: On retention of maxillofacial prosthesis. Odontol Revy 1972; 22: 317-325.

[5]. Ronse J A, Chalian V A.: Fabrication of hollow extraoral prosthesis to enhance retention. J. Prosthet Dent 1985; 53: 557-564.

[6]. Padmanabhan TV, Mohamed K, Parameswari D, Nitin SK. Prosthetic rehabilitation of an orbital and facial defect: A clinical report. J Prosthodont 2012;21(3):200-4

[7]. Jani RM, Schaaf NG. An evaluation of facial prostheses. J Prosthet Dent 1978;39:546-50.

[8]. Kanter JC. The use of RTV silicones in maxillofacial prosthetics. J Prosthet Dent 1970;24:646-53.

[9]. Vashisht R, Kaur H, Prithviraj DR. Finger prosthesis using silicone elastomer: A novel methodology. Int. J Contemp Dent Med Rev 2015;Article ID: 280115. doi: 10.15713/ins.ijcdmr.73.

[10]. Parel SM. Diminishing dependence on adhesives for retention of facial prosthesis. J. Prosthet. Dent. 1980;43:552-60.

[11]. Jani RM, Schaaf NG. An evaluation of facial prostheses. J Prosthet Dent 1978;39:546-50.

\section{Figures}

Fig. 1.An orbital defect.

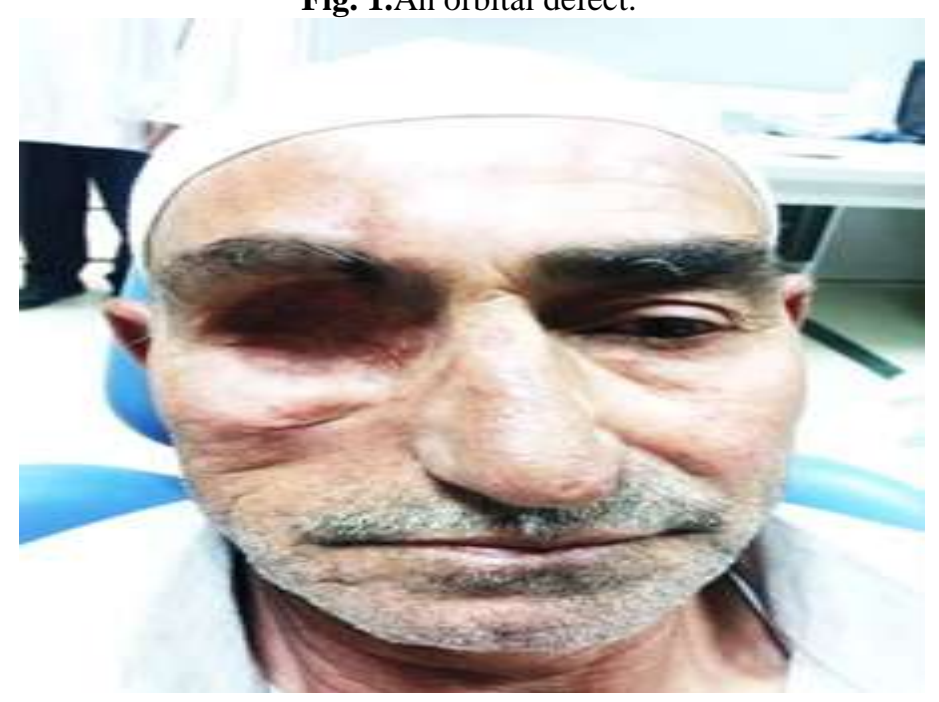

Fig. 2. Facial impression with alginate and defect impression

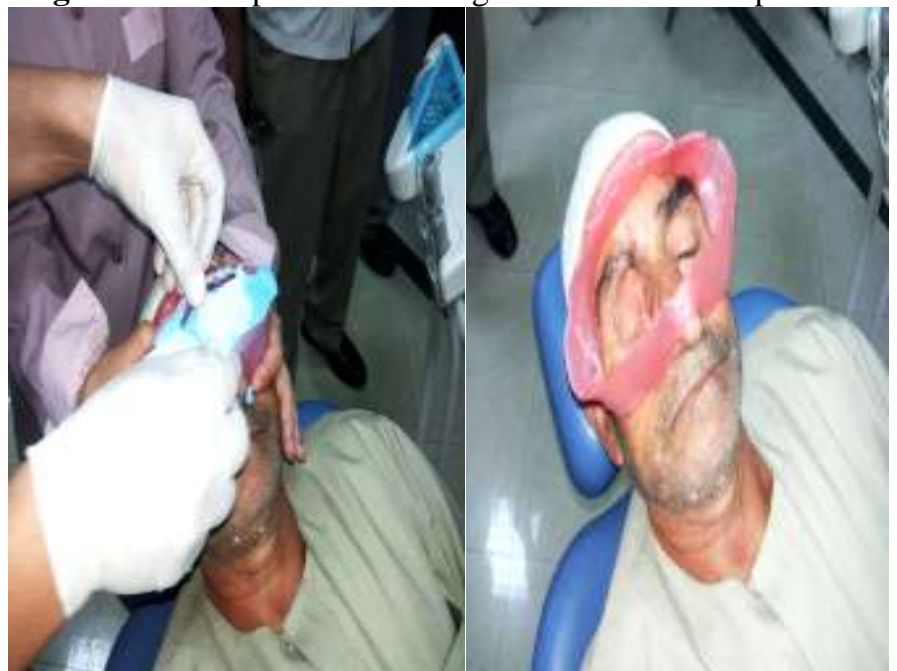

Fig. 3. Moulage of the defect. 


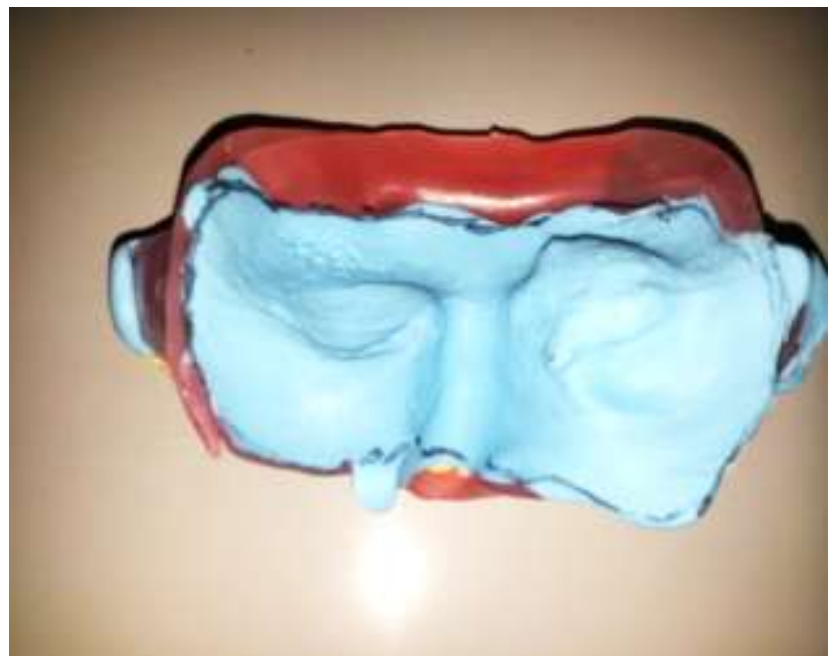

Fig. 4. Orientation marks.

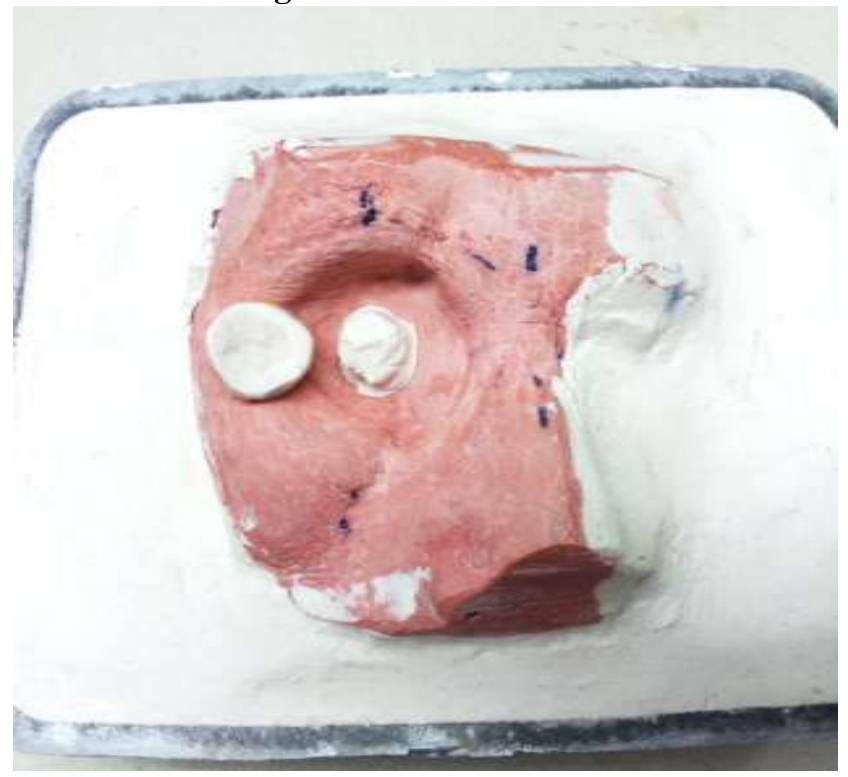

Fig. 5. Wax pattern trial.

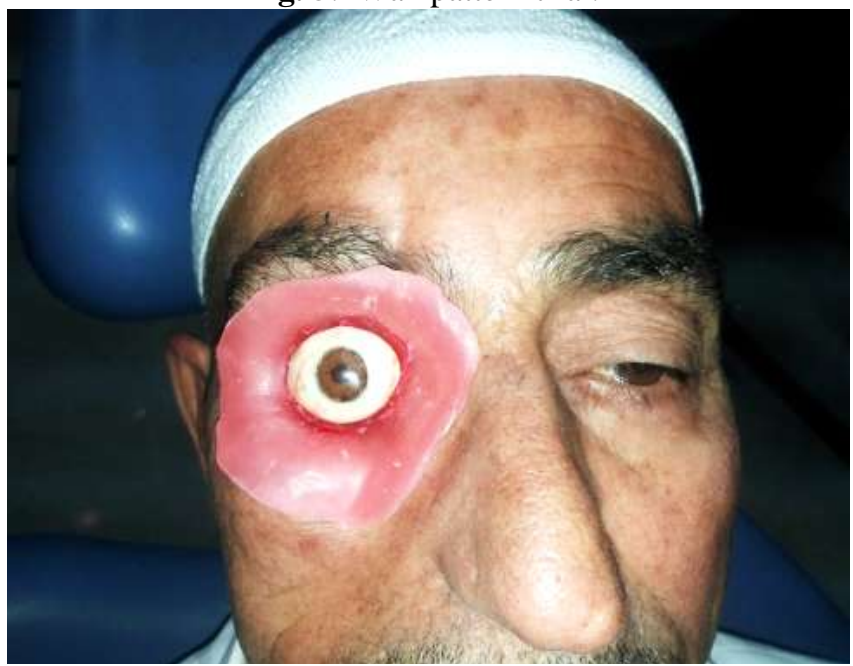

Fig. 6. Acrylic framework trial 


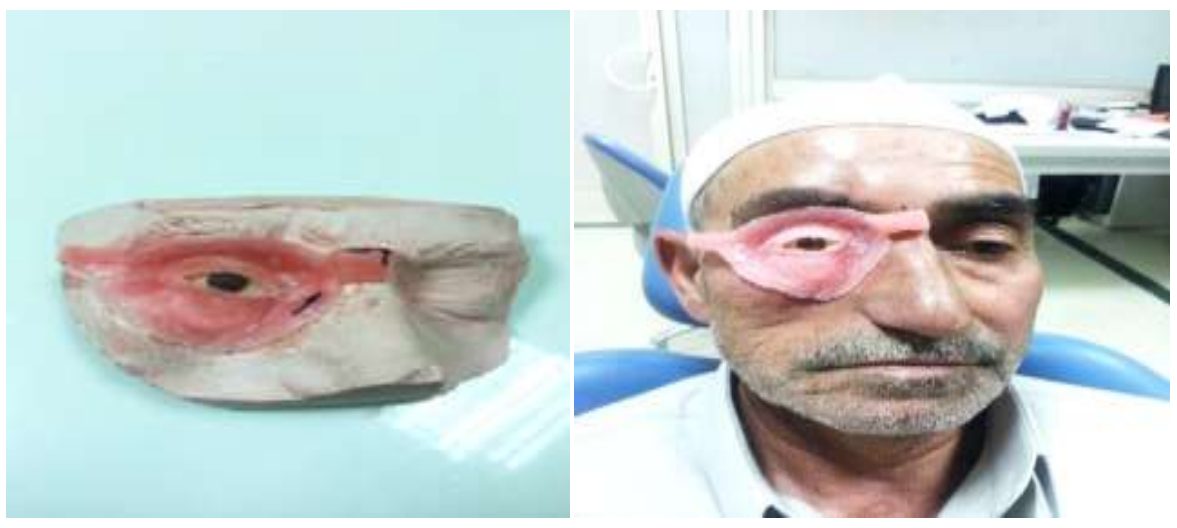

Fig. 7. Acrylic frame trial with the Spectacle

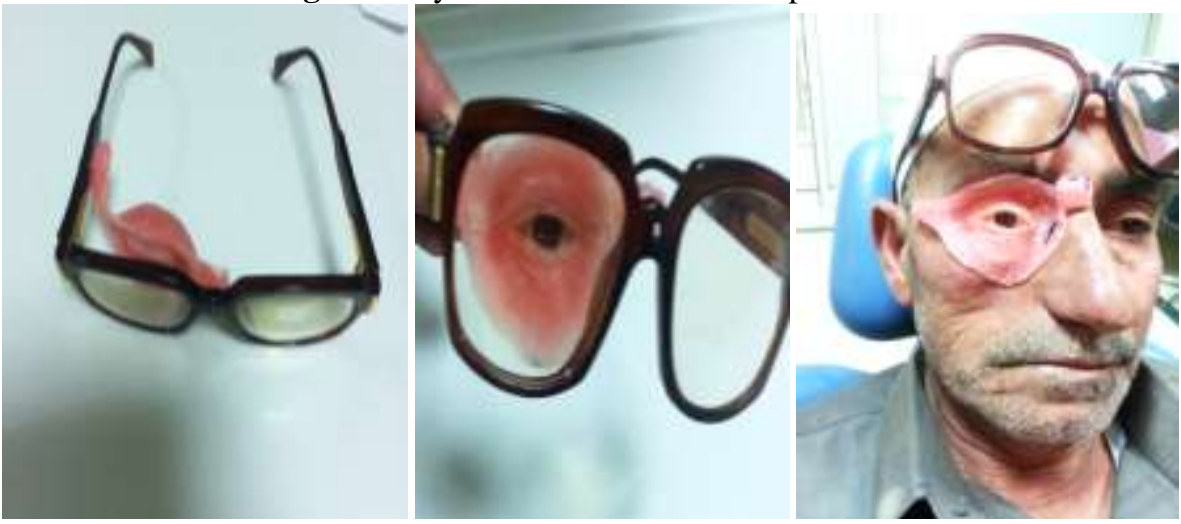

Fig. 8. Intrinsic coloration of silicone

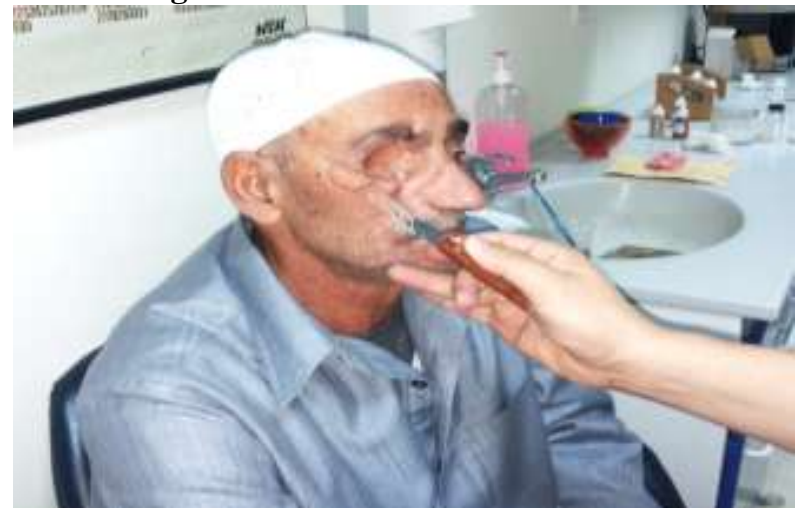

Fig. 9. Silicone Packing and curing

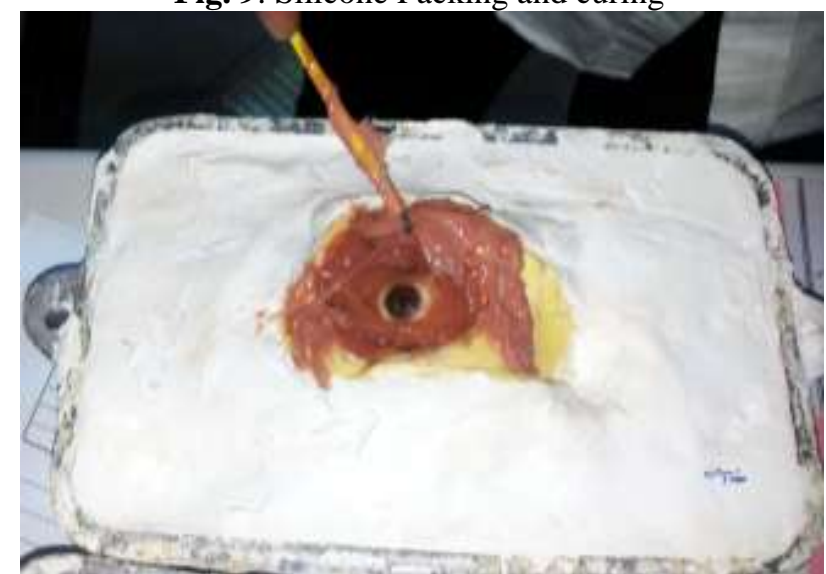

Fig. 10. Extrinsic coloration of the silicone prosthesis 


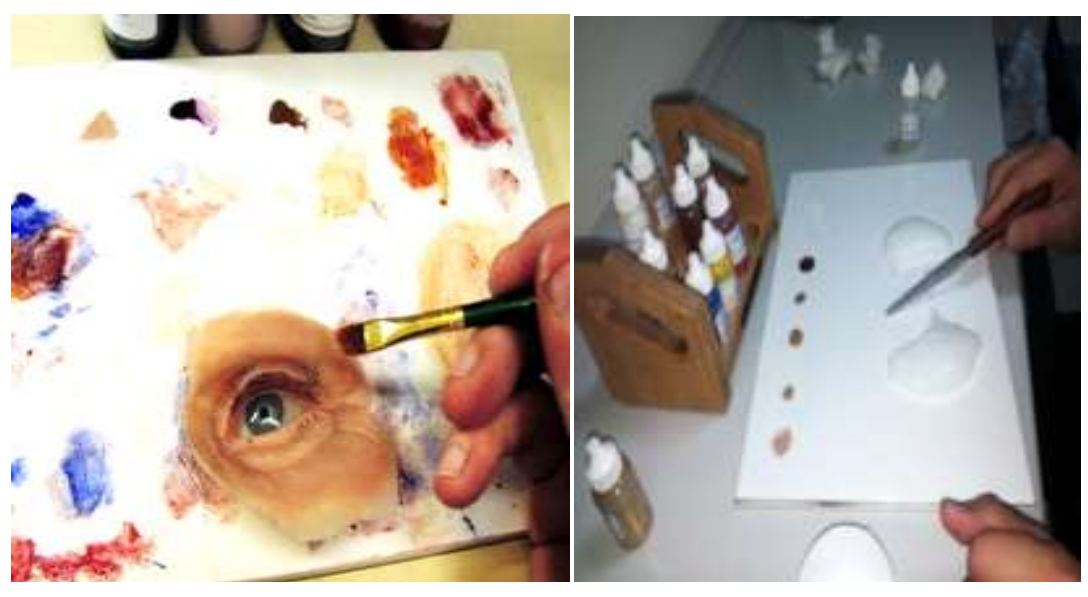

Fig. 11. Insertion of eyelashes

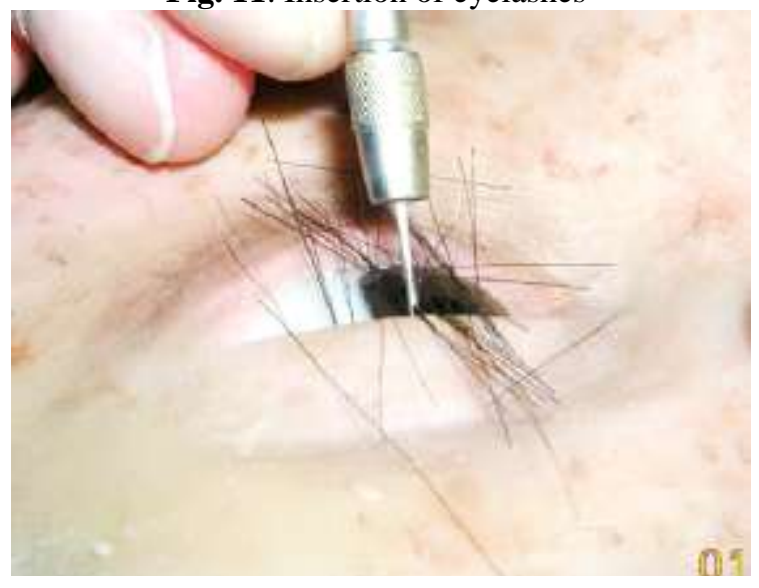

Fig. 12. Orbital prosthesis attachedto the spectacle framework

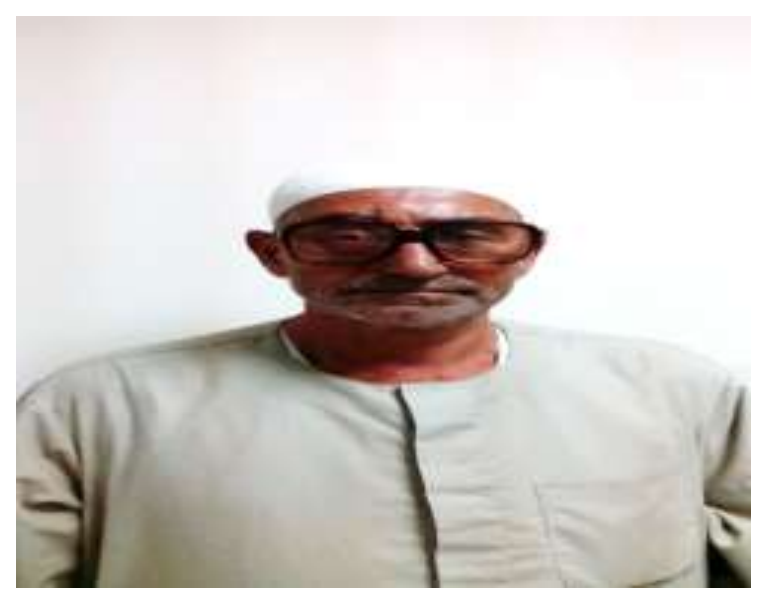

